\title{
SADDLE POINT APPROXIMATION FOR THE DISTRIBUTION OF THE SUM OF INDEPENDENT RANDOM VARIABLES
}

\author{
ROBERT LUGANNANI* AND \\ STEPHEN RICE, ${ }^{*}$ University of California, San Diego
}

\begin{abstract}
In the present paper a uniform asymptotic series is derived for the probability distribution of the sum of a large number of independent random variables. In contrast to the usual Edgeworth-type series, the uniform series gives good accuracy throughout its entire domain. Our derivation uses the fact that the major components of the distribution are determined by a saddle point and a singularity at the origin. The analogous series for the probability density, due to Daniels, depends only on the saddle point. Two illustrative examples are presented that show excellent agreement with the exact distributions.
\end{abstract}

SADDLE POINT APPROXIMATION; SUM OF INDEPENDENT RANDOM VARIABLES; UNIFORM ASYMPTOTIC SERIES

\section{Introduction}

The problem of calculating the probability $Q_{N}(y)$ that the sum

$$
Y=v_{1}+v_{2}+\cdots+v_{N}
$$

of $N$ independent, identically distributed, random variables will exceed $y$ has been extensively studied. In technical applications, where numerical values are of prime importance, a number of methods of determining $Q_{N}(y)$ have been used. A common one is to use the fast Fourier transform which works well when $Q_{N}(y)$ is not too small or when $N$ is not too large. Another is to evaluate numerically the integral

$$
Q_{N}(y)=\frac{1}{2 \pi} \int_{-\infty}^{\infty} e^{-i u y}[g(u)]^{N} d u /(i u),
$$

where the characteristic function $g(u)$ is the Fourier transform of the probability density $p_{1}(v)$ of the typical $v_{j}$ in (1), and the path of integration is indented

Received 9 February 1978; revision received 20 April 1979.

* Postal address: Department of Applied Physics and Information Science, University of California, San Diego, La Jolla, CA 92093, U.S.A.

This research was sponsored by the Air Force Office of Scientific Research, Air Force Systems Command, USAF, under Grant No. AFOSR 74-2689. 
downwards at the origin. This method is capable of high accuracy but the integral often converges slowly and a detailed study of the asymptotic behavior of $g(u)$ may be required.

Here we present an asymptotic series for $Q_{N}(y)$ that we have found useful in calculations associated with signal detection problems (see the remarks made by Olver on the philosophy of using asymptotic series for numerical calculations [6], p. 519). Our series takes into account, in the manner of uniform asymptotic series, the mutual effect of the pole of the integrand at $u=0$ and the 'principal saddle point' $u_{0}$ on the imaginary $u$-axis. Although $u_{0}$ does not exist for all densities $p_{1}(v)$, it does exist in many cases of practical interest. The question of existence has been studied by Daniels [5].

The saddle point $u_{0}$ has been used in a number of investigations. It appears, in effect, in the study of large deviations. See Petrov [7], Chapter 8 where work by Cramér, Saulis and others is described. Daniels [5] has given an asymptotic series for the probability density $p_{N}(y)$ of $(1)$ based on $u_{0}$, whose integration provides an approach alternative to ours (cf. Part (d) of Section 3). Roberts [9] has used $u_{0}$ to deal with communication problems and it appears implicitly in the Chernoff bound [4].

The series for $Q_{N}(y)$ is described in Section 2. In Section 3 several remarks are made about the series and the existence of $u_{0}$ is discussed briefly. Section 4 gives sufficient conditions for our series to be truly asymptotic. Estimation of the error is discussed in Section 5 and illustrated by examples in Sections 6 and 7. In Section 6 an example in which $p_{1}(v)$ is an exponential density is discussed and in Section 7 the uniform density is examined. Finally, in Section 8 the results are used to compare values of $Q_{N}(y)$ obtained from our series with those obtained from formulas given by Cramér and Saulis.

\section{The asymptotic series for $Q_{N}(y)$}

The integral for $Q_{N}(y)$ can be written as

$$
Q_{N}(y)=\frac{1}{2 \pi} \int_{-\infty}^{\infty} e^{N[\phi(i u)-i u r]} d u /(i u),
$$

where $\exp [\phi(i u)]=g(u)$ and $r=y / N$. Our main result is the asymptotic series

$$
\begin{gathered}
Q_{N}(y) \sim \frac{1}{2} \operatorname{erfc}\left(\sqrt{ }-f_{0}\right)+\sum_{n=0}^{\infty}\left(A_{n}-B_{n}\right), \\
f_{0}=N \phi\left(i u_{0}\right)-i u_{0} y, \\
\frac{1}{2} \operatorname{erfc}(x)=\pi^{-\frac{1}{2}} \int_{x}^{\infty} \exp \left(-t^{2}\right) d t=(2 \pi)^{-\frac{1}{2}} \int_{x \sqrt{ } 2}^{\infty} \exp \left(-t^{2} / 2\right) d t,
\end{gathered}
$$


where $A_{n}$ is given below by (9) for $n=0,1,2$, and $B_{n}$ is given by (8). The series (3) is derived in the appendix. In (4) $u_{0}$ is the principal saddle point of $\exp [N \phi(i u)-i u y]$ mentioned in the introduction. If this saddle point exists it lies on the imaginary $u$-axis and is the root of

$$
\frac{d \phi(i u)}{d u}-i r=0
$$

which becomes zero when $y=\bar{y}=E Y$.

Equation (3) is a special case of a class of 'uniform asymptotic series' for integrals containing a large parameter (Bleistein [2], van der Waerden [11], and Rice [8]). The large parameter is $N$ and the uniformity is with respect to $r$.

In calculating $A_{n}$ and $B_{n}$ it is convenient to set $t_{0}=i u_{0}$ where $t_{0}$ is the appropriate real root of

$$
\frac{d}{d t} \phi(t)-r=0
$$

It turns out that $t_{0}$ is positive when $y>\bar{y}$, negative when $y<\bar{y}$, and zero when $y=\bar{y}$. In terms of $t_{0}(4)$ becomes

$$
f_{0}=N \phi\left(t_{0}\right)-t_{0} y,
$$

where it can be shown that $f_{0} \leqq 0$ with equality only when $t_{0}=0$. The sign of $\sqrt{ }-f_{0}$ is taken to be the same as that of $t_{0}$.

The term $B_{n}$ is the $n$th term in the asymptotic series for $\frac{1}{2} \operatorname{erfc}\left(\sqrt{ }-f_{0}\right)$,

$$
B_{n}=\frac{1}{2}\left(-\pi f_{0}\right)^{-\frac{1}{2}} f_{0}^{-n}\left(\frac{1}{2}\right)_{n} \exp \left(f_{0}\right),
$$

where $(\alpha)_{0}=1$ and $(\alpha)_{n}=\alpha(\alpha+1) \cdots(\alpha+n-1)$ when $n>0$. The term $A_{n}$ is the $n$th term in the asymptotic series obtained using the classical saddle point method to expand the integral (2) for $Q_{N}(y)$ about $u_{0}$. As $(y-\bar{y}) / N$ increases, the distance between $u_{0}$ and the pole at $u=0$ increases, and the classical asymptotic series $Q_{N}(y) \sim \sum A_{n}$ becomes increasingly accurate. The presence of the complementary error function and the terms $B_{n}$ increase the accuracy of (3) for small values of $(y-\bar{y}) / N$.

The first three values of $A_{n}$ are

$$
\begin{aligned}
A_{0}= & \mu[2 \pi N]^{-\frac{1}{2}} \exp \left(f_{0}\right), \\
A_{1}= & -3 A_{0} N^{-1}\left[\frac{1}{3} \mu^{2}+\mu \theta_{3}+\frac{1}{2}\left(5 \theta_{3}^{2}-2 \theta_{4}\right)\right], \\
A_{2}= & 15 A_{0} N^{-2}\left[\frac{1}{5} \mu^{4}+\mu^{3} \theta_{3}+\frac{1}{2} \mu^{2}\left(7 \theta_{3}^{2}-2 \theta_{4}\right)\right. \\
& +\frac{1}{4} \mu\left(42 \theta_{3}^{3}-28 \theta_{3} \theta_{4}+4 \theta_{5}\right) \\
& \left.+\frac{1}{8}\left(231 \theta_{3}^{4}-252 \theta_{3}^{2} \theta_{4}+56 \theta_{3} \theta_{5}+28 \theta_{4}^{2}-8 \theta_{6}\right)\right],
\end{aligned}
$$


where

$$
\begin{aligned}
\phi^{(n)} & =\left[(d / d t)^{n} \phi(t)\right]_{t_{0}}=\left[i^{-n}(d / d u)^{n} \phi(i u)\right]_{u_{0}}, \\
\theta_{n} & =\phi^{(n)} /\left(n !\left[\phi^{(2)}\right]^{n / 2}\right), \\
\mu & =1 /\left(t_{0}\left[\phi^{(2)}\right]^{\frac{1}{2}}\right) .
\end{aligned}
$$

Here $\phi^{(2)}$ is positive and $\phi^{(n)}$ can be interpreted as the $n$th cumulant of the 'associated' density (see Daniels [5], pages 639 and 640)

$$
\begin{aligned}
& \hat{p}_{1}(v)=e^{v t_{0}} p_{1}(v) / g\left(u_{0}\right), \\
& g\left(u_{0}\right)=\int_{-\infty}^{\infty} e^{v t_{0}} p_{1}(v) d v .
\end{aligned}
$$

The characteristic function for $\hat{p}_{1}(v)$,

$$
E e^{i x v}=g\left(u_{0}+x\right) / g\left(u_{0}\right),
$$

will be used in Section 4 .

\section{Remarks concerning the asymptotic series for $Q_{N}(y)$}

(a) General values of $n$. $A_{n}$ can be calculated for general values of $n$ by using a recurrence relation for the coefficients in the classical asymptotic series. Thus, from Equation (103) of [8], (changing $n$ to $j$ )

$$
A_{j}=A_{0} N^{-i} \sum_{n=0}^{2 i}(-\mu)^{2 j-n} \sum_{m=0}^{n} d_{m, n}(-2)^{m+j}\left(\frac{1}{2}\right)_{m+j},
$$

where $d_{m, n}$ is computed step by step from

$$
d_{m+1, n+1}=\frac{1}{n+1} \sum_{k=1}^{n-m+1} k \theta_{k+2} d_{m, n-k+1}, \quad 0 \leqq m \leqq n
$$

starting from $d_{00}=1$ and $d_{0 n}=0$ for $n>0$. For $m=0$ and $n \geqq 1$ we get $d_{1 n}=\theta_{n+2}$ and for $m=n, d_{n n}=\theta_{3}^{n} / n$ !. It is often convenient to use $\left(\frac{1}{2}\right)_{m+j}=$ $\Gamma\left(m+j+\frac{1}{2}\right) / \pi^{\frac{1}{2}}$ in (13).

Daniels' series [5] for the probability density $p_{N}(y)=-(d / d y) Q_{N}(y)$ is

$$
p_{N}(y) \sim\left[2 \pi N \phi^{(2)}\right]^{-\frac{1}{2}} \exp \left(f_{0}\right)\left[1-\frac{3}{2 N}\left(5 \theta_{3}^{2}-2 \theta_{4}\right)+\cdots\right] .
$$

Equation (15) can be written as $p_{N}(y) \sim \sum \tilde{A}_{j}$ where

$$
\begin{aligned}
& \tilde{A}_{0}=\left[2 \pi N \phi^{(2)}\right]^{-\frac{1}{2}} \exp \left(f_{0}\right) \\
& \tilde{A}_{j}=\tilde{A}_{0} N^{-i} \sum_{m=1}^{2 j} d_{m, 2 j}(-2)^{m+j}\left(\frac{1}{2}\right)_{m+j}
\end{aligned}
$$


and $d_{m, 2 j}$ is given by (14). Replacing $A_{0}$ by $\tilde{A}_{0}$ and setting $\mu=0$ in the expression (13) for $A_{j}$ gives $\tilde{A}_{j}$. For example, $\tilde{A}_{1}$ and $\tilde{A}_{2}$ can be obtained from (9) by setting $\mu=0$ in $A_{1}$ and $A_{2}$.

(b) The case $y=\bar{y}$. When $y=\bar{y}, t_{0}$ is zero, and $A_{n}$ and $B_{n}$ are infinite, but the limit of $A_{n}-B_{n}$ remains finite as $y \rightarrow \bar{y}$. In this case a classical saddle point analysis gives

$$
\begin{aligned}
Q_{N}(\bar{y}) \sim & \frac{1}{2}+(2 \pi N)^{-\frac{1}{2}}\left[-\theta_{3}+N^{-1}\left(\frac{35}{2} \theta_{3}^{3}-15 \theta_{3} \theta_{4}+3 \theta_{5}\right)\right. \\
& -\frac{15}{2} N^{-2}\left(\frac{3003}{20} \theta_{3}^{5}-231 \theta_{3}^{3} \theta_{4}+63 \theta_{3} \theta_{4}^{2}+63 \theta_{3}^{2} \theta_{5}\right. \\
& \left.\left.-14 \theta_{4} \theta_{5}-14 \theta_{3} \theta_{6}+2 \theta_{7}\right)+\cdots\right] .
\end{aligned}
$$

It can be verified that the terms in (17) agree with corresponding ones in the Edgeworth series ([1], No. 26.2.48) for the case $y=\bar{y}$.

(c) Another form of (3). The form (3) of the asymptotic series for $Q_{N}(y)$ is convenient for calculations when $N$ is fixed and $y$ varies. A different form, useful in analyzing the errors, can be obtained by introducing $C_{n}$ defined by

$$
A_{n}-B_{n}=C_{n} N^{-n-\frac{1}{2}} \exp \left(N \gamma_{0}\right) \Gamma\left(n+\frac{1}{2}\right) / \pi,
$$

where $\gamma_{0}=\gamma\left(u_{0}\right)=f_{0} / N$ and

$$
\gamma(u)=\phi(i u)-i u r
$$

Then (3) becomes

$$
Q_{N}(y) \sim \frac{1}{2} \operatorname{erfc}\left(\sqrt{-N \gamma_{0}}\right)+\frac{1}{\pi} e^{N \gamma_{0}} \sum_{n=0}^{\infty} C_{n} \Gamma\left(n+\frac{1}{2}\right) N^{-n-\frac{1}{2}} .
$$

The structure of $A_{n}-B_{n}$ shows that $C_{n}$ does not depend explicitly on $N$ although there is an implicit dependence via $r$.

(d) Integration of the Daniels series. An interesting question arises regarding the accuracy of our asymptotic series for $Q_{N}(y)$ compared with that of the series obtained by integrating Daniels' series (15) for $p_{N}(y)$. It appears difficult to give an answer in the general case because of the complexity of the integration. However, some insight can be obtained by examining the exponential distribution discussed later in Section 6. From the results given there it can be shown that the Daniels series is

$$
p_{N}(y) \sim p_{N \text { ex }}(y)\left[N ! / N^{N} e^{-N} \sqrt{2 \pi N]}\left(1-\frac{1}{12 N}+\frac{1}{288 N^{2}}+\cdots\right),\right.
$$

where $p_{N \text { ex }}(y)$ is the exact density $y^{N-1} e^{-y} /(N-1) !, y>0$. When we integrate and take the first two terms, for example, we get

$$
\hat{Q} A(2)=Q_{e x}\left[N ! / N^{N} e^{-N} \sqrt{2 \pi N]}\left(1-\frac{1}{12 N}\right),\right.
$$


where $Q_{\text {ex }}$ is the exact value (38) of $Q_{N}(y)$. $\hat{Q} A(2)$ is to be compared with our

$$
Q A(2)=\frac{1}{2} \operatorname{erfc}\left(\sqrt{ }-f_{0}\right)+\left(A_{0}-B_{0}\right)+\left(A_{1}-B_{1}\right)
$$

given by (45). The 'relative error' $\mathscr{C}$ of $Q A(2)$ is plotted in Figure 1 for the case $N=5$. For $N=5$ the relative error of $\hat{Q} A(2)$ can be shown to be $\hat{\mathscr{E}}=-0.00016$ when $y>5$. Comparison with Figure 1 shows that $\mathscr{E}$ and $\hat{\mathscr{E}}$ are of the same order of magnitude in this particular case.

This example suggests the conjecture that integration of Daniels' series and our asymptotic series for $Q_{N}(y)$ both give approximations to $Q_{N}(y)$ that are in error by the same order of magnitude.

(e) Existence of $u_{0}$. We conclude this section with some remarks regarding the existence of $u_{0}$ that are based on Daniels' work [5]. Let $p_{1}(v)$ be zero outside of $a \leqq v \leqq b$ where $a$ or $b$ may be infinite. It can be shown that $u_{0}$ exists for every value of $y / N$ between $a$ and $b$ if the integral

$$
\int_{a}^{b} e^{t v} p_{1}(v) d v
$$

exists for all real values of $t$. Examples are:

(i) Finite $a$ and $b$.

(ii) $a=-\infty, b=\infty$ and $p_{1}(v) \leqq A \exp \left(-|v|^{1+\varepsilon}\right)$ where $\varepsilon>0$ and $A$ is a constant.

When the integral does not exist for all values of $t$, the question becomes more complicated:

(iii) For $a=0, b=\infty, p_{1}(v)=A \exp \left(-v^{1-\varepsilon}\right)$ and $0<\varepsilon<1, u_{0}$ exists when $0 \leqq y \leqq \bar{y}$, but not when $y>\bar{y}$.

(iv) When $a=0, b=\infty, p_{1}(v)=A v^{\alpha-1}(1+v)^{-\beta} e^{-v}$ and $\alpha>0, u_{0}$ exists for the entire range $0 \leqq y \leqq \infty$ if $\beta \leqq \alpha+1$. When $\beta>\alpha+1, u_{0}$ exists only if $0 \leqq y / N \leqq 1 /(\beta-\alpha-1)$. In both cases $u_{0}$ runs from $i \infty$ to $-i$ as $y$ runs over the range for which $u_{0}$ exists.

(v) When $p_{1}(v)=\frac{1}{2} \exp (-|v|)$ there are two saddle points on the imaginary axis. As $y$ runs from $-\infty$ to $+\infty$, the principal saddle point $u_{0}$ runs from $+i$ to $-i$.

\section{Sufficient conditions for the series to be asymptotic}

Here sufficient conditions are given for the series (3) to be asymptotic in $1 / N$ when $r=y / N$ is fixed. We consider only the case $y>\bar{y}$ in detail. The analysis for the case $y<\bar{y}$ is very similar and will not be repeated. For $y>\bar{y}, t_{0}$ is positive and $u_{0}=-i t_{0}$ lies on the negative imaginary $u$-axis.

Suppose that $u_{0}$ has been determined for some fixed value of $r$, and let $P$ denote the straight line path $\operatorname{Im}\left(u-u_{0}\right)=0$ joining $u_{0} \pm \infty$ and passing through $u_{0}$. Let the characteristic function $g(u)$ of $p_{1}(v)$ satisfy the conditions: 
(i) $g(u)$ is analytic throughout a strip $-t_{0}-\varepsilon \leqq \operatorname{Im}(u) \leqq \varepsilon$ where $\varepsilon$ is some positive constant.

(ii) Positive constants $\alpha, c_{0}$ and $c_{1}$ exist such that $|g(u)|<c_{0} /|u|^{\alpha}$ when $|u|>c_{1}$ on the path $P$.

These conditions allow us to displace the path of integration in the integral (2) for $Q_{N}(y)$ down to $P$. After making this displacement, the expression (4) for $f_{0}$, and the relation $g(u)=\exp [\phi(i u)]$ are used to rewrite (2) as

$$
Q_{N}(y)=\frac{\exp \left(f_{0}\right)}{\pi} \operatorname{Re} \int_{0}^{\infty} \exp \left\{N\left[\phi\left(i u_{0}+i x\right)-\phi\left(i u_{0}\right)-i x r\right]\right\} d x /\left(i u_{0}+i x\right)
$$

where $x=u-u_{0}$. In (21)

$$
\exp \left[\phi\left(i u_{0}+i x\right)-\phi\left(i u_{0}\right)\right]=g\left(u_{0}+x\right) / g\left(u_{0}\right),
$$

where, from (12), the right-hand side of (22) is the characteristic function $E \exp (i v x)$ of the associated density $\hat{p}_{1}(v)$. Since $x$ is real in (21), $\lg \left(u_{0}+\right.$ $x) / g\left(u_{0}\right) \mid \leqq 1$ with equality only at $x=0$ because $\hat{p}_{1}(v)$ has no lattice component (as a consequence of (ii)). Therefore in (21)

$$
\operatorname{Re}\left[\phi\left(i u_{0}+i x\right)-\phi\left(i u_{0}\right)-i x r\right] \leqq 0
$$

with equality only at $x=0$. Furthermore, Condition (i) shows that $\phi\left(i u_{0}+i x\right)-$ $\phi\left(i u_{0}\right)$ - ixr can be expanded in a power series in $x$ that converges in the neighborhood of $x=0$.

The preceding discussion and the fact that the contribution to $Q_{N}(y)$ of the region around $u_{0}$ is $\sum A_{n}$ (as already mentioned in connection with (9)) shows that Conditions (i) and (ii) are sufficient to guarantee that

$$
Q_{N}(y) \sim \sum_{n=0}^{\infty} A_{n}
$$

as $N \rightarrow \infty$ (see Olver [6], Chapter 4, Section 6). Subtracting the known asymptotic series

$$
\frac{1}{2} \operatorname{erfc}\left(\sqrt{ }-f_{0}\right) \sim \sum_{n=0}^{\infty} B_{n}
$$

completes the proof that (3) is indeed an asymptotic series for $Q_{N}(y)$ when $y>\bar{y}$ and $r=y / N$ is fixed.

\section{Error analysis}

Let the conditions of Section 4 be satisfied and rewrite (2) as

$$
Q_{N}(y)=\operatorname{Re} \frac{1}{\pi} \int_{u_{0}}^{\infty+i 0} e^{N \gamma(u)} d u /(i u),
$$


where $\gamma(u)=\phi(i u)-i u r$. Let the right-hand branch of the steepest descent path of $\exp [\gamma(u)]$ from $u_{0}$ end at the sink $u=s_{0}$ where $\gamma\left(s_{0}\right)=-\infty$. Then

$$
Q_{N}(y)=I\left(u_{0}, s_{0}\right)+I\left(s_{0}, \infty+i 0\right),
$$

where $I\left(u_{1}, u_{2}\right)$ denotes the integral in (25) with limits $u_{1}, u_{2}$.

The sink $s_{0}$ occurs at a zero of the characteristic function $g(u)$, but it may shift from one zero to another as $r=y / N$ changes. The path of steepest descent from $u_{0}$ is given by $\operatorname{Im}\left[\gamma\left(u_{0}\right)-\gamma(u)\right]=0$. If the path cannot be determined easily by analysis it can be traced step by step by starting at $u_{0}+\Delta$ and using

$$
u_{l+1}=u_{l}-\left|\gamma^{\prime}(u)\right| \Delta / \gamma^{\prime}(u),
$$

where $\Delta$ is the step length and $\gamma^{\prime}(u)=d \gamma(u) / d u$.

It is convenient to regard the right-hand side of (3) as the asymptotic expansion of $I\left(u_{0}, s_{0}\right)$ and $I\left(s_{0}, \infty+i 0\right)$ as an exponentially small correction term. This point of view is helpful in explaining the fact that an asymptotic series may sometimes appear to be more accurate than it actually is (Olver [6], p. 95).

Let $m \geqq 1$ and define the partial sum $Q A(m)$ by

$$
Q A(m) \triangleq \frac{1}{2} \operatorname{erfc}\left(\sqrt{ }-f_{0}\right)+\sum_{n=0}^{m-1}\left(A_{n}-B_{n}\right) .
$$

Then the error in $Q A(m)$ is

$$
\begin{aligned}
Q A(m)-Q_{N}(y) & =\left[Q A(m)-I\left(u_{0}, s_{0}\right)\right]+\left[-I\left(s_{0}, \infty+i 0\right)\right] \\
& =E P_{m}+E S,
\end{aligned}
$$

where the principal error $E P_{m}$ and the exponentially small error $E S$ are defined by the quantities within the brackets:

$$
\begin{gathered}
E P_{m} \triangleq Q A(m)-\operatorname{Re} \frac{1}{\pi} \int_{u_{0}}^{s_{0}} e^{N \gamma(u)} d u /(i u), \\
E S \triangleq-\operatorname{Re} \frac{1}{\pi} \int_{s_{0}}^{\infty+i 0} e^{N \gamma(u)} d u /(i u) .
\end{gathered}
$$

$E S$ depends only on $N$. It can be zero in some cases and greater than $E P_{m}$ in others. A bound for $\left|E P_{m}\right|$ can be obtained by modifying Olver's error bound ([6], p. 89) for asymptotic series so as to take $\operatorname{erfc}\left(\sqrt{ }-f_{0}\right)$ into account. If $\left(A_{m}-B_{m}\right)$ is not zero it can be shown that

$$
\left|E P_{m}\right| \leqq\left[N /\left(N-\sigma_{m}\right)\right]^{m+\frac{1}{2}}\left|A_{m}-B_{m}\right|,
$$

where $\sigma_{m}$ is the supremum of a complicated function of $m, r$ and $u$ along the 
steepest path from $u_{0}$ to $s_{0}$ :

$$
\begin{aligned}
& \sigma_{m}=\sup _{\tau \in(0, \infty)}\left[\tau^{-1} \ln \left|F_{m}(\tau) /\left(C_{m} \tau^{m-\frac{1}{2}}\right)\right|\right] \\
& \tau=\gamma_{0}-\gamma(u), \\
& F_{m}(\tau)=\frac{1}{2}\left(-\gamma_{0} \tau\right)^{-\frac{1}{2}}\left(1-\tau / \gamma_{0}\right)^{-1}-\operatorname{Re}\left(\frac{1}{i u} \frac{d u}{d \tau}\right)+\sum_{n=0}^{m-1} C_{n} \tau^{n-\frac{1}{2}}
\end{aligned}
$$

Here $C_{n}$ is defined by $(18)$ and $\gamma_{0}=\gamma\left(u_{0}\right)$.

\section{Example-The exponential distribution}

(a) Asymptotic series. The characteristic function for the one-sided density $p_{1}(v)=e^{-v}, v>0$ is

$$
\begin{aligned}
g(u) & =\int_{0}^{\infty} e^{i u v-v} d v \\
& =1 /(1-i u)
\end{aligned}
$$

and

$$
\phi(i u)=\ln g(u)=-\ln (1-i u) .
$$

The saddle point equation (6) gives $u_{0}=-i t_{0}$ where $t_{0}=1-r^{-1}$ with $r=y / N$. The quantities needed to calculate $A_{n}$ and $B_{n}$ are

$$
\begin{aligned}
& f_{0}=N(\ln r-r+1), \quad \phi^{(n)}=(n-1) ! r^{n} . \\
& \theta_{n}=1 / n, \quad \mu=1 /(r-1),
\end{aligned}
$$

and (8) and (9) give

$$
\begin{array}{ll}
A_{0}=\mu(2 \pi N)^{-\frac{1}{2}} e^{f_{0}} & B_{0}=\frac{1}{2}\left(-\pi f_{0}\right)^{-\frac{1}{2}} e^{f_{0}}, \\
A_{1}=A_{0} N^{-1}\left(-\mu^{2}-\mu-\frac{1}{12}\right), & B_{1}=B_{0} /\left(2 f_{0}\right), \\
A_{2}=A_{0} N^{-2}\left(3 \mu^{4}+5 \mu^{3}+\frac{25}{12} \mu^{2}+\frac{1}{12} \mu+\frac{1}{288}\right), & B_{2}=3 B_{1} /\left(2 f_{0}\right) .
\end{array}
$$

When $y=\bar{y}=N, r$ is $1, \mu$ is infinite, and it is necessary to use the Edgeworth series

$$
Q_{N}(y) \sim \frac{1}{2}+(2 \pi N)^{-\frac{1}{2}}\left[-\frac{1}{3}-\frac{1}{N} \frac{1}{540}+\frac{1}{N^{2}} \frac{25}{6048}+\cdots\right] .
$$

The exact expression for $Q_{N}(y)$ is

$$
Q_{N}(y)=e^{-y} \sum_{n=0}^{N-1} y^{n} / n !
$$


(b) Error analysis. An examination of the path of steepest descent of $\exp [N \gamma(u)]$, where $\gamma(u)=-\ln (1-i u)-i u r$, from $u_{0}$ shows that the sink $s_{0}$ occurs at $s_{0}=\pi / r-i \infty$, and consequently the exponentially small error $E S$ is zero. To illustrate the bound (31) for $E P_{m}$ consider the case $r=y / N=2$ and $m=1$. Numerical evaluation of (32) at points along the path between $u_{0}$ and $s_{0}$ shows that the supremum occurs at $\tau=6.94$. It has the value $\sigma=0.085$ and (31) becomes

$$
\left|E P_{1}\right| \leqq[N /(N-0 \cdot 085)]^{\frac{3}{2}}\left|A_{1}-B_{1}\right| .
$$

Calculations for $N=5$ and $y=r N=10$ show that

$$
\begin{aligned}
Q_{5}(10) & =0 \cdot 02925 \cdots, \\
E P_{1} & =Q A(1)-Q_{5}(10)=-2 \cdot 18(-5), \\
A_{1}-B_{1} & =-2 \cdot 58(-5) .
\end{aligned}
$$

Therefore (39) gives $\left|E P_{1}\right| \leqq 2 \cdot 65(-5)$ which is slightly larger than $\left|E P_{1}\right|=$ $2 \cdot 18(-5)$.

\section{Example-The uniform distribution}

The characteristic function corresponding to $p_{1}(v)=\frac{1}{2}$ for $-1<v<1$ is $g(u)=$ $\sin u / u$, and $\phi(i u)=\ln g(u)=\ln (\sin u / u)$. In order to calculate (3) we need the values of

$$
\phi(t)=\ln (\sinh t / t)
$$

and its derivatives at the real root $t_{0}$ of

$$
\cosh t-t^{-1}-r=0 \text {. }
$$

Equation (41) can be solved by starting with $t_{0} \approx 1 /(1-r)$ and using the Newton-Raphson method. Differentiation of (40) gives

$$
\begin{aligned}
& (d / d t)^{2} \phi(t)=-\operatorname{csch}^{2} t+t^{-2} \\
& (d / d t)^{3} \phi(t)=\left(2 \operatorname{csch}^{3} t\right) \cosh t-2 t^{-3}
\end{aligned}
$$

and so on. For $t$ real we also have

$$
(d / d t)^{n} \phi(t)=(-)^{n-1}(n-1) ! 2 \sum_{l=1}^{\infty} \operatorname{Re}(t+i \pi l)^{-n}
$$

which is useful when $n$ is large and the recursion relation (13) is used to calculate $A_{n}$. For the error calculations the exact expression

$$
Q_{N}(y)=\frac{1}{2^{N} N !} \sum_{k=0}^{K}(-)^{k}\left(\begin{array}{l}
N \\
k
\end{array}\right)(N-y-2 k)^{N}
$$

was used. Here $K$ is the largest integer in $(N-y) / 2$. 
A trial calculation was made using $N=4$ and $y=3 \cdot 2$. In this case the smallest term in (3) is $A_{3}-B_{3}=-2 \cdot 8(-6)$ and the error made in stopping with this term is $Q A(4)-Q_{4}(3 \cdot 2)=4 \cdot 9(-6)$, i.e.,

$$
E P_{4}+E S=4 \cdot 9(-6)
$$

This is small compared to the exact value $Q_{4}(3 \cdot 2)=1066 \cdot 7(-6)$.

(a) The error ES. For the uniform distribution, the function $\gamma(u)$ appearing in the definition (30) of $E S$ is $\gamma(u)=\ln (\sin u / u)-i u r$. When the path of steepest descent from $u_{0}$ is calculated by using $\gamma^{\prime}(u)=\cot u-u^{-1}-i r$ in the step-by-step formula (27) it is found that $s_{0}$ is one of the zeros, say $l \pi$, of $\sin u / u$. If $r$ is between 0 and a number slightly larger than $0 \cdot 7, l$ is 1 . For $r=0 \cdot 8, l$ is 2 , and for $r=0 \cdot 9, l$ is 5 . From (30) and $r=y / N$,

$$
E S=\frac{1}{\pi} \int_{l \pi}^{\infty}\left(\frac{\sin u}{u}\right)^{N} \frac{\sin u y}{u} d u .
$$

For $N=4, y=3 \cdot 2$ we get $r=0 \cdot 8, l=2$ and the value of $E S$ calculated from (44) is $-2 \cdot 1(-6)$. This is an appreciable fraction of the total error $4 \cdot 9(-6)$ stated above for our partial sum $Q A(4)$. Incidentally, when $l=0$ in (44) the integral is equal to $Q_{N}(y)$.

\section{Comparison with other approximations}

Here we compare our first two partial sums

$$
\begin{aligned}
& Q A(1)=\frac{1}{2} \operatorname{erfc}\left(\sqrt{ }-f_{0}\right)+\left(A_{0}-B_{0}\right), \\
& Q A(2)=Q A(1)+\left(A_{1}-B_{1}\right),
\end{aligned}
$$

with other approximations to $Q_{N}(y)$. The comparison is based on the relative error defined by

$$
\begin{array}{ll}
\mathscr{E}=\left(Q_{\text {ap }}-Q_{\text {ex }}\right) / Q_{\text {ex }}, & y>\bar{y}, \\
\mathscr{E}=\left[\left(1-Q_{\text {ap }}\right)-\left(1-Q_{\text {ex }}\right)\right] /\left(1-Q_{\text {ex }}\right), & y<\bar{y},
\end{array}
$$

where $Q_{a p}$ is the approximation and $Q_{\text {ex }}$ is the exact value $Q_{N}(y)$.

Figure 1 shows $|\mathscr{E}|$ for approximations to $Q_{5}(y)$ when the individual density is $p_{1}(v)=\exp (-v), v>0$ (Section 6$)$. The sign of $\mathscr{E}$ is indicated by the + or on the curve. A portion of $Q A(2)$ around $y=\bar{y}=5$ has been omitted to reduce the clutter. The 'Edge ${ }_{2}$ ' curve is calculated from an Edgeworth series ([1], No. 26.2.48) in which the last term depends on the cumulants $\kappa_{2}=\sigma^{2}=1, \kappa_{3}=2$, $\kappa_{4}=6$ of $v$ and the third and fifth derivatives of $\exp \left(-x^{2} / 2\right)$ where

$$
\begin{aligned}
x & =(y-\bar{y}) /(\sigma \sqrt{ } N), \\
& =(y-5) / 2 \cdot 236, \quad N=5 .
\end{aligned}
$$




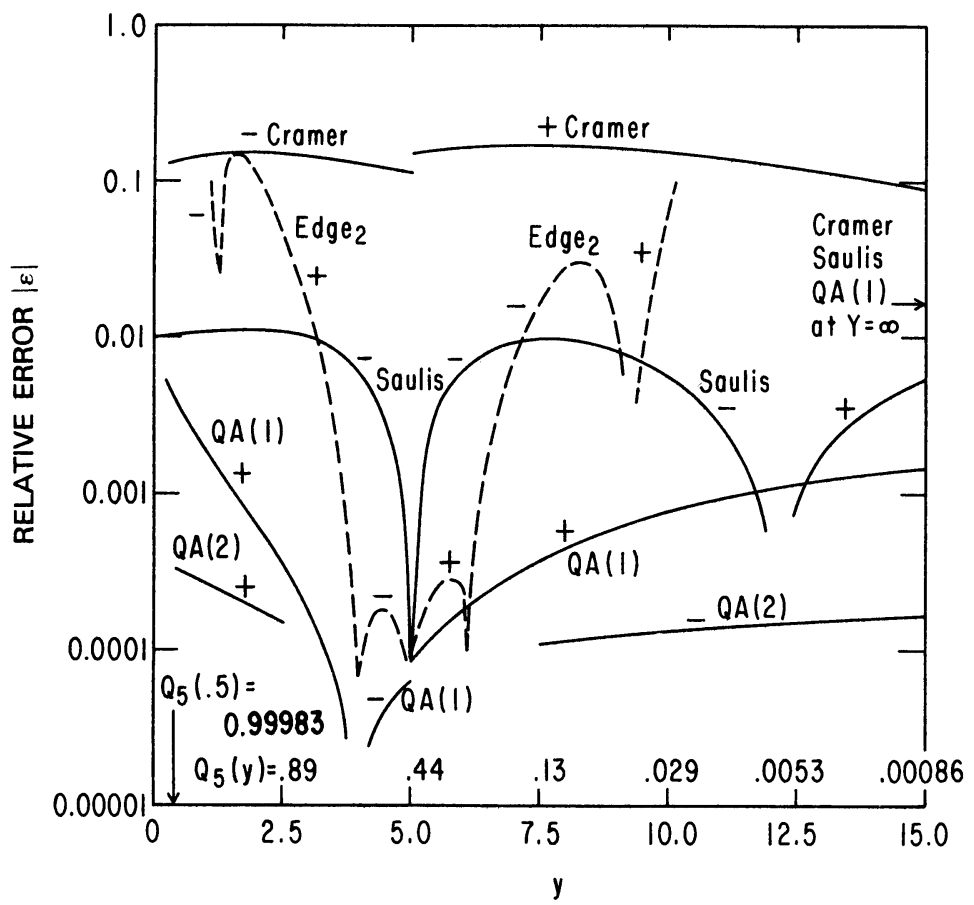

Figure 1

Relative error of various approximations to $Q_{5}(y)$ when $p_{1}(v)=\exp (-v), v>0$.

For $y>\bar{y}$ the 'Cramér' curve is calculated from the approximation

$$
Q_{N}(y) \approx[1-\Phi(x)] \exp \left[f_{0}+\frac{1}{2} x^{2}\right]
$$

obtained by deleting the 'order of' term in the equation for $Q_{N}(y)$ presented in [7], p. 219. Here $1-\Phi(x)=\frac{1}{2} \operatorname{erfc}(x / \sqrt{ } 2)$ and $x$ is given by (47). A similar result holds when $y<\bar{y}$.

In (48) we have made use of the fact that Cramér's function $\lambda(z)$ is related to our $f_{0}$ by

$$
\left(x^{3} / \sqrt{ } N\right) \lambda(x / \sqrt{ } N)=f_{0}+\frac{1}{2} x^{2} .
$$

The 'Saulis' curve for $y>\bar{y}$ is calculated in much the same way as the 'Cramér' curve by using an expression given by Petrov ([7], p. 249) which represents the first two terms in a general series given by Saulis [10].

Figure 2 shows $|\mathscr{E}|$ for approximations to $Q_{5}(y)$ when $p_{1}(y)=\frac{1}{2},|v|<1$, the uniform distribution (Section 7). The Edge ${ }_{2}$ curve is calculated from the same general formulas as for the exponential distribution but now the cumulants are $\kappa_{2}=\sigma^{2}=\frac{1}{3}, \kappa_{3}=0, \kappa_{4}=-\frac{2}{15}$ and $x=y /(\sigma \sqrt{ } N)=y \sqrt{\frac{3}{5}}$. It turns out that, because $\kappa_{3}=0$, the two-term Saulis formula reduces to Cramér's result.

It is seen that $Q A(1)$ and $Q A(2)$ do quite well over the entire range of $y$. 


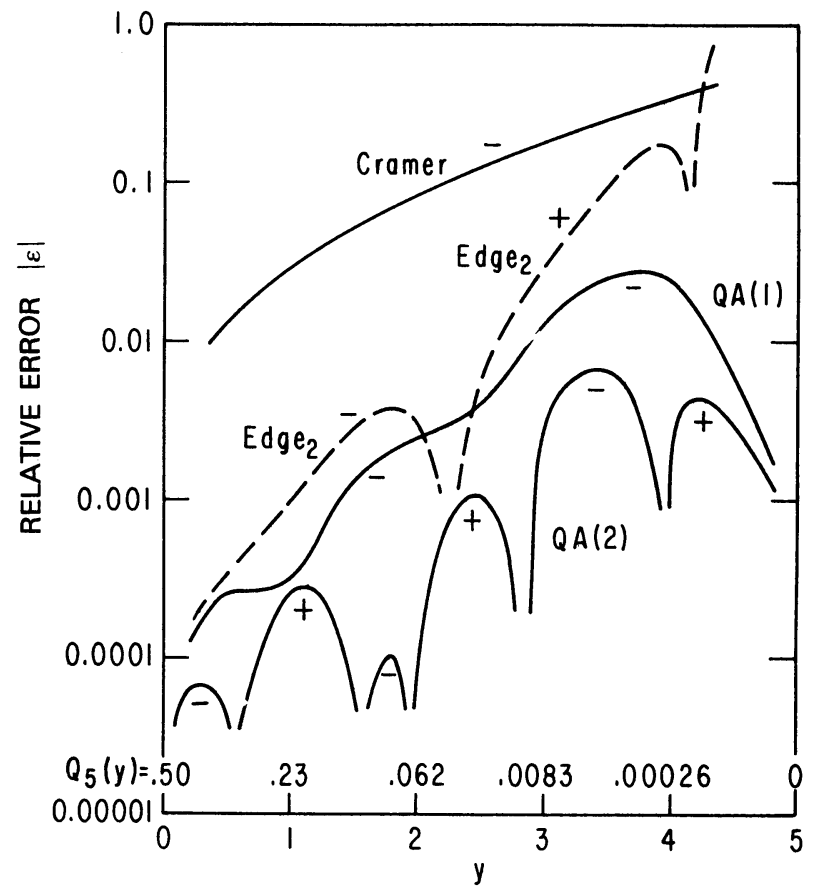

Figure 2

Relative error of various approximations to $Q_{5}(y)$ when $p_{1}(v)=\frac{1}{2},|v|<1$.

\section{Appendix. Uniform asymptotic series for integrals}

The integral (2) for $Q_{N}(y)$ is a special case (with a change of notation) of the integral

$$
J=\int_{L^{\prime}} t^{\lambda-1} g(t) e^{x h(t)} d t
$$

where $x$ is large and positive. Methods associated with the names of Bleistein and Ursell for expanding (50) in a 'uniform asymptotic series' have been discussed in [8].

(a) General comments. First assume that the term $\exp [x h(t)]$ in (50) has $\mu$ simple saddle points (where the first derivative of $h(t)$ vanishes but the second does not) and that $\lambda$ is not a positive integer. The saddle points and the origin lie within a relatively small 'critical' region in the $t$-plane through which the path $L^{\prime}$ passes. The functions $g(t)$ and $h(t)$ are analytic throughout the critical region, $g(0) \neq 0, h(0)=0$ and $t=0$ is not a saddle point.

In the critical region $h(t)$ behaves like a polynomial of degree $\mu+1$. Let $v$ be a new variable such that $F(v)=h(t)$ where $F(v)$ is a polynomial of degree $\mu+1$ in $v$, and $v$ is nearly proportional to $t$ in the critical region. The choice of $F(t)$ is 
discussed in [8]. This change of variable carries (50) into

$$
J=\int_{L} v^{\lambda-1} f(v) e^{x F(v)} d v
$$

where $L$ in the $v$-plane corresponds to $L^{\prime}$ in the $t$-plane. Let $t_{1}, t_{2}, \cdots, t_{\mu}$ be the saddle points in the $t$-plane and $v_{1}, v_{2}, \cdots, v_{\mu}$ the corresponding ones in the $v$-plane. Deforming $L$ into paths of steepest descent and considering the separate contributions of the saddle points leads to an asymptotic expansion of the form

$$
\begin{gathered}
J \sim \sum_{l=0}^{\mu} V_{l}(x)\left(p_{0 l}+p_{1 l} x^{-1}+p_{2 l} x^{-2}+\cdots\right), \\
V_{l}(x)=\int_{L} v^{l+\lambda-1} e^{x F(v)} d v,
\end{gathered}
$$

where $V_{l}(x)$ is regarded as a tabulated or easily computed function. It turns out that $p_{n l}$ does not depend on $L^{\prime}$ or $L$, and by choosing suitable paths we can get a set of equations that can be solved for the $p_{n l}$ 's.

(b) The one-saddle-point case. For illustration consider the case $\mu=1$. A treatment of this case which differs somewhat from the following one is given in Appendix $\mathrm{F}$ of [8], and entirely different treatments are given by Bleistein in Sections 6 and 7 of [2] and by van der Waerden [11]. See also the excellent discussion of uniform asymptotic expansions given in Chapter 9 of Bleistein and Handelsman [3]. We seek an expansion of the form (52) with $\mu=1$ when $V_{0}(x)$ and $V_{1}(x)$ are given by (53) in which $F(v)$ is a second-degree polynomial. A convenient choice is

$$
F(v)=v^{2}-2 v_{1} v
$$

Let $L_{1}^{\prime}$ be the path of steepest descent that has $t_{1}$ as its highest point. A classical saddle-point expansion about $t_{1}$ gives

$$
\begin{aligned}
J_{1} & =\int_{L_{i}^{\prime}} t^{\lambda-1} g(t) e^{x h(t)} d t \\
& \sim \exp \left[x h\left(t_{1}\right)\right] \sum_{n=0}^{\infty} \alpha_{1 n} x^{-n-\frac{1}{2}},
\end{aligned}
$$

where the $\alpha_{1 n}$ 's can be calculated from the derivatives of $g(t)$ and $h(t)$ at $t_{1}$ (see, for example, (103) of [8]). Similarly, from (52) and (53), $J_{1}$ can also be expressed as

$$
J_{1} \sim \sum_{l=0}^{1}\left[V_{l}(x)\right]_{1}\left(p_{0 l}+p_{1 l} x^{-1}+\cdots\right)
$$




$$
\begin{aligned}
{\left[V_{l}(x)\right]_{1} } & =\int_{L_{1}} v^{l+\lambda-1} e^{x F(v)} d v \\
& \sim \exp \left[x F\left(v_{1}\right)\right] \sum_{m=0}^{\infty} \beta_{1 l m} x^{-m-\frac{1}{2}},
\end{aligned}
$$

where $L_{1}$ is the path that has $v_{1}$ as its highest point.

Putting (57) in (56), equating coefficients of $x^{-n-\frac{1}{2}}$ in (55) to those in (56), and using

$$
\beta_{110}=\beta_{100} v_{1}
$$

gives a set of relations, the $n$th of which is

$$
p_{n 1}=-\frac{p_{n 0}}{v_{1}}+\frac{\alpha_{1 n}}{\beta_{110}}-\frac{1}{\beta_{110}} \sum_{k=0}^{n-1}\left(\beta_{1,0, n-k} p_{k 0}+\beta_{1,1, n-k} p_{k 1}\right),
$$

where the summation is omitted when $n$ is 0 .

Another set of relations can be obtained by treating the singularity at the origin in somewhat the same way as the saddle point. If the singularity is a branch point we take $L_{0}^{\prime}$ and $L_{0}$ to be loops enclosing the branch cuts running out from the origins. If the singularity is a pole we take $L_{0}^{\prime}$ and $L_{0}$ to be small circles around the origins. In any case

$$
\begin{gathered}
J_{0}=\int_{L_{0}^{\prime}} t^{\lambda-1} g(t) e^{x h(t)} d t \sim \sum_{n=0}^{\infty} \alpha_{0 n} x^{-n-\lambda}, \\
{\left[V_{l}(x)\right]_{0}=\int_{L_{0}} v^{l+\lambda-1} e^{x F(v)} d v \sim \sum_{m=0}^{\infty} \beta_{0 l m} x^{-m-l-\lambda} .}
\end{gathered}
$$

Equating the two asymptotic series for $J_{0}$ leads to

$$
p_{n 0}=\frac{\alpha_{0 n}}{\beta_{000}}-\frac{1}{\beta_{000}} \sum_{k=0}^{n-1}\left(\beta_{0,0, n-k} p_{k 0}+\beta_{0,1, n-k} p_{k 1}\right) \text {. }
$$

Equations (59) and (62) can be used to calculate $p_{n 0}$ and $p_{n 1}$ step by step, starting with $p_{00}=\alpha_{00} / \beta_{000}$ from (62).

(c) Application to $Q_{N}(y)$. For $Q_{N}(y)$ we consider the special case in which $\lambda=0, g(t)=1, \mu=1, t_{1}$ is real, and $L^{\prime}$ runs from $-i \infty$ to $+i \infty$ with an indentation to the right at $t=0$ (see Section 9 of [8] and Bleistein [2]). When $F(v)$ is defined by (54), (53) gives

$$
V_{0}(x)=i \pi \operatorname{erfc}\left(v_{1} x^{\frac{1}{2}}\right), \quad V_{1}(x)=i(\pi / x)^{\frac{1}{2}} \exp \left(-x v_{1}^{2}\right) .
$$

If $L_{0}^{\prime}$ and $L_{0}$ are taken to be small circles about the origin,

$$
J_{0}=2 \pi i, \quad\left[V_{0}(x)\right]_{0}=2 \pi i, \quad\left[V_{1}(x)\right]_{0}=0,
$$

and consequently all of the $\alpha_{0 n}, \beta_{00 m}, \beta_{01 m}$ are zero except $\alpha_{00}=2 \pi i$ and 
$\beta_{000}=2 \pi i$. The recurrence relation (62) then gives

$$
p_{00}=1, \quad p_{n 0}=0, \quad n \geqq 1 .
$$

The $\alpha_{1 n}$ 's are defined by

$$
\int_{L_{i}^{\prime}} t^{-1} e^{x h(t)} d t \sim e^{x h\left(t_{1}\right)} \sum_{n=0}^{\infty} \alpha_{1 n} x^{-n-\frac{1}{2}}
$$

where $L_{1}^{\prime}$ runs upward through $t_{1}$. Taking $L_{1}$ to run from $-i \infty$ to $+i \infty$ through $v_{1}$ makes $\left[V_{l}(x)\right]_{1}=V_{l}(x), l=0,1$. Noting that the asymptotic series for $V_{1}(x)$ consists of only the leading term shows that $\beta_{11 m}$ in (57) is 0 except for $\beta_{110}=i \pi^{\frac{1}{2}}$. Similarly, the asymptotic series for $V_{0}(x)$ leads to

$$
\beta_{10 m}=i \pi^{\frac{1}{2}}(-)^{m}\left(\frac{1}{2}\right)_{m} v_{1}^{-2 m-1}, \quad m=0,1,2 \cdots .
$$

Note that $\beta_{110}=v_{1} \beta_{100}$ as it should according to (58). The recurrence relation (59) then gives

$$
p_{n 1}=\left(\alpha_{1 n}-\beta_{10 n}\right) / \beta_{110} .
$$

Inserting the values of $p_{n 0}$ and $p_{n 1}$ in the series (52) leads to

$$
\int_{L^{\prime}} t^{-1} e^{x h(t)} d t \sim i \pi \operatorname{erfc}\left(v_{1} x^{\frac{1}{2}}\right)+\exp \left(-x v_{1}^{2}\right) \sum_{n=0}^{\infty}\left(\alpha_{1 n}-\beta_{10 n}\right) x^{-n-\frac{1}{2}} .
$$

Since $h\left(t_{1}\right)=F\left(v_{1}\right)=-v_{1}^{2}$ this series has essentially the same form as the series (3) for $Q_{N}(y)$.

\section{References}

[1] Abramowitz, M. And Stegun, I. A. (1972) Handbook of Mathematical Functions. Dover, New York.

[2] BLEISTEIN, N. (1966) Uniform asymptotic expansions of integrals with stationary point near algebraic singularity. Comm. Pure Appl. Math. 19, 353-370.

[3] Bleistein, N. And Handelsman, R. A. (1975) Asymptotic Expansion of Integrals. Holt, Rinehart and Winston, New York.

[4] CHERnOFF, H. (1952) A measure of asymptotic efficiency for tests of a hypothesis based on the sum of observations. Ann. Math. Statist. 23, 493-507.

[5] Daniels, H. E. (1954) Saddlepoint approximations in statistics. Ann. Math. Statist. 25, 631-650.

[6] Olver, F. W. J. (1974) Asymptotics and Special Functions. Academic Press, New York.

[7] Petrov, V. V. (1973) Sums of Independent Random Variables, trans. A. A. Brown. Springer-Verlag, New York.

[8] RICE, S. O. (1968) Uniform asymptotic expansions for saddle point integrals-Applications to a probability distribution occurring in noise theory. Bell Syst. Tech. J. 47, 1971-2013.

[9] RoBERTS, J. B. (1972) Distribution of the response of linear systems to Poisson distributed random impulses. J. Sound and Vibration 24, 23-34.

[10] Saulis, L. (1969) Asymptotic expansions of probabilities of large deviations (in Russian). Litovsk. Mat. Sb. 9, 605-625.

[11] Van Der Waerden, B. L. (1951) On the method of saddle points. Appl. Sci. Res. B2, $33-45$. 